\title{
Assessment of Livin Expression in Childhood B-lineage Acute Lymphoblastic Leukemia Patients
}

Hosneia Khalaf Akl ${ }^{1}$, Amina Mohamed Talaat ${ }^{1}$, Mervat Abd Allah Hesham², Sara Mohamed Mohamed Ibrahim ${ }^{* 1}$

Departments of ${ }^{1}$ Clinical Pathology and ${ }^{2}$ Pediatrics, Faculty of Medicine, Zagazig University, Egypt

*Corresponding Author: Sara Mohamed Mohamed Ibrahim, Email: saraabdelwahab1980@ hotmail.com

\begin{abstract}
Background: When it comes to childhood acute lymphoblastic leukemia (ALL) patients, Livin is related with good prognostic characteristics.

Objective: The aim of the work was to assess livin expression in childhood B-lineage acute lymphoblastic leukemia to determine its significance.

Patients and Methods: From September 2018 and September 2019, at Zagazig University Hospitals Clinical Pathology and Pediatric departments, our case control trial was conducted on 50 subjects, patients' group ( 25 cases of newly diagnosed ALL) and control group (25 healthy children). Assessment of livin expression was done using real time polymerase chain reaction (PCR).

Results: There was statistically significant difference between the age of livin positive and livin negative cases (the mean age of livin positive cases was $6.2 \pm 3$ years while the mean age of livin negative cases was $12.3 \pm 1.4$ years). Mean livin expression level was significantly higher in age group $<10$ than in age group group $\geq 10$ years $(294.53 \pm 154.03$ in age group $<10$ years versus $31 \pm 12.37$ in age group $\geq 10$ ). In livin negative group there were 3 patients with leucocytic count $<50.000 / \mathrm{mm} 3$ at diagnosis while 5 patients with leucocytic count $\geq 50.000 / \mathrm{mm} 3$ or more at diagnosis compared to 14 and 3 patients among livin positive group respectively $(\mathrm{p}=0.025)$. Regarding age, sex, WBCs, and risk stratification, there were statistically significant differences between the livin negative and positive groups.
\end{abstract}

Conclusion: It could be concluded that livin was associated with favorable prognostic factors among ALL patients: (age $<10$ years, female patients, WBCs $<50,000 / \mathrm{mm} 3$ ).

Keywords: Livin expression, Acute Lymphoblastic Leukemia.

\section{INTRODUCTION}

Around $80 \%$ of all pediatric leukemias are acute lymphoblastic leukemia (ALL), making it the most frequent form of childhood cancer ${ }^{(\mathbf{1})}$.

Apoptosis is a self-sustaining process in which a large number of genes are activated, expressed, and regulated to cause programmed cell death in order to eliminate undesirable or defective cells ${ }^{(2)}$.

There is a class of apoptotic regulators, known as Inhibitor apoptotic Proteins (IAPs), that promote cancer cells' resistance to apoptosis, notably in cancer treatment and chemotherapy. In order to reestablish the apoptotic response to proapoptotic stimuli, disrupting the binding of IAPs to their functional partners is a viable method ${ }^{(3)}$.

Cell cycle and proliferation are affected as well as apoptosis when Livin/ML-IAP/BIRC7 is present, making it an important player in these processes. Livin, which is over-expressed in a number of cancers, has an anti-apoptotic effect mediated by the direct suppression of caspase 3, but also caspases 7 and 9 as well as DIABLO $^{(4)}$.

Different isoforms of livin may play different functions in apoptosis. Truncated proteins are formed when Livin isoforms are cleaved. To be effective, the shortened form of the protein, known as livin (t- livin), must also have the ability to cause cell death. Thus, livin has both the ability to shield csll cells from cell death and the ability to promote it once it is cleaved ${ }^{(5)}$.
More specifically, livin was found to be related with positive prognostic characteristics in children with acute lymphoblastic leukemia (ALL) ${ }^{(6)}$.

It was the goal of this study to assess the significance of livin expression in childhood B -lineage acute lymphoblastic leukemia.

\section{PATIENTS AND METHODS}

This case-control trial study included a total of 25 cases of newly diagnosed All and 25 healthy children served as control, attending at Departments of Clinical Pathology and Pediatrics, Zagazig University Hospitals, Egypt. This study was conducted between July 2020 to July 2021.

The included 50 subjects were divided into two groups; Control Group: Included 25 apparently healthy children with mean age $9.1 \pm 3.6$ years; $60 \%$ of them were males (15 males) and $40 \%$ of them were females (10 females), and Case Group: Included 25 newly diagnosed ALL patients who attended the Pediatric Hematology and Oncology unit with mean age $8.1 \pm 3.9$ years; $(56 \%)$ of them were males (14 males), and (44\%) of them were females (11females).

\section{Ethical Consideration:}

This study was ethically approved by Zagazig University's research ethics committee. Written informed consent of all the participants' parents was

This article is an open access article distributed under the terms and conditions of the Creative Commons Attribution (CC BY-SA) license (http://creativecommons.org/licenses/by/4.0/) 
obtained and submitted them to Zagazig University (ZU-IRB\#3598). We adhered to the Helsinki Declaration, the ethical norm of the World Medical Association for human testing.

Inclusion Criteria: De novo cases of ALL (didn't receive any chemotherapy before), and age: $<18$ years, of both sexes.

Exclusion Criteria: Cases having malignant disorder other than acute lymphoblastic leukemia, and patients exhibiting an immunophenotype of $\mathrm{T}$ - cell acute lymphoblastic leukemia.

Patients were classified according to their risk into standard risk and high risk based on age and WBC count at diagnosis: Standard risk: age from 1 to less than 10

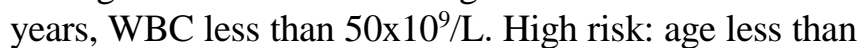
1 year or more than 10 years, WBC more than $50 \times 10^{9} / \mathrm{L}$.

All participants were subjected to history taking, clinical examination and laboratory assessment of livin expression using real time polymerase chain reaction (PCR).

\section{Statistical analysis}

The collected data were coded, processed and analyzed using the SPSS (Statistical Package for Social Sciences) version 22 for Windows ${ }^{\circledR}$ (IBM SPSS Inc, Chicago, IL, USA). Numbers and percentages are used to represent data (percent) or mean $\pm \mathrm{SD}$. Different qualitative factors were examined using the Chi square (X2) test. If the significant probability was less than 0.05 , the threshold for statistical significance, the results were considered statistically significant and highly significant. $\mathrm{P}$ value $<0.05$ was considered significant.

\section{RESULTS}

(Table 1) shows demographic and clinical characteristics of control and case groups, with significant statistically differences between them regarding lymphadenopathy, splenomegaly and hepatomegaly.

(Table 2) shows mean \pm SD of CBC characteristics of control and case groups with statistically highly significant differences as regard Hemoglobin (HB), white blood cells (WBC) and platelets (PLT).

(Table 3) shows mean \pm SD of liver, kidney functions and livin in control and cases. No statistically significant differences between cases and controls as regard any of the tests except LDH and livin which means show highly significant increase in case than control $(\mathrm{P}<0.001)$.

(Table 4) shows there was statistically significant increase in livin negative than positive cases regarding mean age, statistically significant differences between both groups regarding age and FAB distribution and risk stratification, while no significant differences regarding other items.

(Table 5) shows relation between livin expression and laboratory findings: As regard WBC there was statistically significant difference between livin +ve cases and livin -ve cases: in livin -ve cases 3 patients presented with WBCs $<50.000 / \mathrm{mm}^{3}$ while 5 patients presented with WBCs $\geq 50.000 / \mathrm{mm}^{3}$ compared to 14 and 3 among livin $+v e$ cases respectively $(\mathrm{p}=0.025)$. A significant difference existed between the two groupings in terms of $\mathrm{BM}$ blast $(\mathrm{P}=0.05)$. Livin +ve and -ve patients had no statistically significant differences with regard to the following: Hb, PLT; PT; PTT; SGOT; SGPT; SGOT; SGPT; Total bilirubin; TP; Albumin; Urea; LDH; creatinine; and peripheral blast.

(Table 6) shows that mean livin expression was significantly higher in age group $<10$ years than those who are $>10$ years $(\mathrm{p}<0.001)$. Mean livin expression was higher in patients with Pre-B ALL than those of mature progenitor B-ALL $<$ and also significantly higher in standard risk group than high risk group $(\mathrm{P}=0.01)$.

Table (1): Demographic and clinical characteristics of control and cases:

\begin{tabular}{|c|c|c|c|c|c|c|}
\hline \multirow{2}{*}{ Variables } & \multicolumn{2}{|c|}{ Control } & \multicolumn{2}{|c|}{ Case } & \multirow{2}{*}{ Test $* * *$} & \multirow{2}{*}{$P$ value } \\
\hline & \multicolumn{2}{|l|}{$\mathrm{N}=25$} & \multicolumn{2}{|c|}{$\mathrm{N}=\mathbf{2 5}$} & & \\
\hline Age (years) $($ mean \pm SD) & \multicolumn{2}{|c|}{$9.10 \pm 3.60$} & \multicolumn{2}{|c|}{$8.10 \pm 3.90$} & $-0.862 * *$ & 0.393 \\
\hline \multicolumn{7}{|l|}{ Sex } \\
\hline - $\quad$ Male & 14 & $56.0 \%$ & 15 & $60.0 \%$ & \multirow{2}{*}{0.082} & \multirow{2}{*}{1.00} \\
\hline - $\quad$ Female & 11 & $44.0 \%$ & 10 & $40.0 \%$ & & \\
\hline \multicolumn{7}{|l|}{ Lymphadenopathy } \\
\hline - Negative & 2 & $8.0 \%$ & 25 & $100.0 \%$ & \multirow{2}{*}{42.593} & \multirow{2}{*}{$<0.001 *$} \\
\hline - $\quad$ Positive & 23 & $92.0 \%$ & 0 & $0.0 \%$ & & \\
\hline \multicolumn{7}{|l|}{ Splenomegaly } \\
\hline - Negative & 3 & $12.0 \%$ & 25 & $100.0 \%$ & \multirow{2}{*}{39.28} & \multirow{2}{*}{$<0.001^{*}$} \\
\hline - $\quad$ Positive & 22 & $88.0 \%$ & 0 & $0 \%$ & & \\
\hline \multicolumn{7}{|l|}{ Hepatomegaly } \\
\hline - Negative & 4 & $16.0 \%$ & 25 & $100.0 \%$ & \multirow{2}{*}{36.21} & \multirow{2}{*}{$<0.001 *$} \\
\hline - $\quad$ Positive & 21 & $84.0 \%$ & 0 & $0.0 \%$ & & \\
\hline
\end{tabular}

**independent sample $\mathrm{t}$ test $* * *$ Chi square test 
Table (2): Complete Blood Count of control and cases:

\begin{tabular}{|c|c|c|c|c|c|c|}
\hline \multirow{2}{*}{ Variables(mean \pm SD) } & \multicolumn{2}{|c|}{ Control } & \multicolumn{2}{|c|}{ Case } & \multirow{2}{*}{ Test } & \multirow{2}{*}{$P$ value } \\
\hline & \multicolumn{2}{|c|}{$\mathrm{N}=\mathbf{2 5}$} & \multicolumn{2}{|c|}{$\mathrm{N}=25$} & & \\
\hline - $\quad \mathrm{HB}(\mathrm{g} / \mathrm{dl})$ & \multicolumn{2}{|c|}{$12.2 \pm 0.5$} & \multicolumn{2}{|c|}{$8.5 \pm 1.7$} & $-10.641 * *$ & $<0.001 *$ \\
\hline - $\quad \mathrm{WBC}\left(\mathrm{x} 10^{3} / \mathrm{mm}^{3}\right)$ & \multicolumn{2}{|c|}{$8.2 \pm 1.6$} & \multicolumn{2}{|c|}{$37.6 \pm 28.1$} & $-4.134 * * *$ & $<0.001 *$ \\
\hline \multicolumn{7}{|l|}{ WBC } \\
\hline - $\quad<50.000 / \mathrm{mm}^{3}$ & 25 & $59.5 \%$ & 17 & $40.5 \%$ & \multirow{2}{*}{9.524} & \multirow{2}{*}{$0.004 *$} \\
\hline - $\quad \geq 50.000 / \mathrm{mm}^{3}$ & 0 & $0.0 \%$ & 8 & $100.0 \%$ & & \\
\hline & \multicolumn{2}{|c|}{ Mean \pm SD } & \multicolumn{2}{|c|}{ Mean \pm SD } & & \\
\hline - $\quad$ PLT: $\left(\times 10^{3} / \mathrm{mm}^{3}\right)$ & \multicolumn{2}{|c|}{$300.0 \pm 69$} & \multicolumn{2}{|c|}{$61.0 \pm 69.0$} & $-5.620 * * *$ & $<0.001 *$ \\
\hline
\end{tabular}

Table (3): Comparison between controls and cases regarding laboratory findings

\begin{tabular}{|c|c|c|c|c|}
\hline \multirow{2}{*}{ Variable (mean \pm SD) } & Control & Case & \multirow{2}{*}{ Test } & \multirow{2}{*}{$P$ value } \\
\hline & $\mathbf{N}=25$ & $\mathrm{~N}=25$ & & \\
\hline Total bilirubin (mg/dl) & $0.8 \pm 0.2$ & $0.8 \pm 0.2$ & $-0.091 * * *$ & 0.928 \\
\hline Direct bilirubin(mg/dl) & $0.30 \pm 0.08$ & $0.30 \pm 0.09$ & $-0.093 * * *$ & 0.926 \\
\hline Total Protein(g/dl) & $7.0 \pm 0.7$ & $6.6 \pm 0.7$ & $-1.747 * * *$ & 0.087 \\
\hline $\operatorname{Albumin}(\mathrm{g} / \mathrm{dl})$ & $4.0 \pm 0.6$ & $3.9 \pm 0.6$ & $-0.817 * *$ & 0.418 \\
\hline SGOT (U/L) & $26.2 \pm 4.3$ & $28.6 \pm 4.5$ & $1.029 * *$ & 0.309 \\
\hline SGPT(U/L) & $28.5 \pm 5.5$ & $29.9 \pm 4.2$ & $1.029 * *$ & 0.309 \\
\hline PT (second) & $12.0 \pm 0.6$ & $12.6 \pm 0.8$ & $-1.174 * * *$ & 0.241 \\
\hline PTT (second) & $30 \pm 3.0$ & $29.0 \pm 3$ & $-0.980 * *$ & 0.332 \\
\hline LDH (U/L) & $158 \pm 17$ & $310 \pm 37$ & $-6.071 * * *$ & $<0.001 *$ \\
\hline Creatinine (mg/dl) & $0.39 \pm 0.08$ & $0.40 \pm 0.07$ & $-0.675 * * *$ & 0.500 \\
\hline Urea(mg/dl) & $14.5 \pm 3.3$ & $13.6 \pm 3.4$ & $-0.963 * *$ & 0.335 \\
\hline Livin & $179.31 \pm 15.66$ & $0.054 \pm 0.01$ & $-6.065 * * *$ & $<0.001 *$ \\
\hline
\end{tabular}

Table (4): Relation between livin expression and Demographic \& clinicopathological characteristics among livin negative and positive subgroups

\begin{tabular}{|c|c|c|c|c|c|c|}
\hline \multirow{3}{*}{ Variable } & \multicolumn{4}{|c|}{$\begin{array}{l}\text { Livin Expression } \\
\end{array}$} & \multirow{3}{*}{ Test $* *$} & \multirow{3}{*}{$P$ value } \\
\hline & \multicolumn{2}{|c|}{ Negative } & \multicolumn{2}{|c|}{ Positive } & & \\
\hline & $\mathrm{N}=8$ & $\%$ & $\mathbf{N}=17$ & $\%$ & & \\
\hline \multicolumn{7}{|l|}{ Age } \\
\hline - $\quad<10$ & 0 & $0.0 \%$ & 15 & $100.0 \%$ & \multirow{2}{*}{17.64} & \multirow{2}{*}{$<0.001^{*}$} \\
\hline - $\quad \geq 10$ & 8 & $80.0 \%$ & 2 & $20.0 \%$ & & \\
\hline \multicolumn{7}{|l|}{ Sex } \\
\hline - $\quad$ Male & 7 & $50.0 \%$ & 7 & $50.0 \%$ & \multirow{2}{*}{4.723} & \multirow{2}{*}{$0.042 *$} \\
\hline - $\quad$ Female & 1 & $9.1 \%$ & 10 & $90.9 \%$ & & \\
\hline \multicolumn{7}{|l|}{ Lymphadenopathy } \\
\hline - Negative & 0 & $0.0 \%$ & 2 & $100.0 \%$ & \multirow{2}{*}{1.023} & \multirow{2}{*}{1.00} \\
\hline - $\quad$ Positive & 8 & $34.8 \%$ & 15 & $65.2 \%$ & & \\
\hline \multicolumn{7}{|l|}{ Splenomegaly } \\
\hline - Negative & 1 & $33.3 \%$ & 2 & $66.7 \%$ & \multirow{2}{*}{0.003} & \multirow{2}{*}{1.00} \\
\hline - $\quad$ Positive & 7 & $31.8 \%$ & 15 & $68.2 \%$ & & \\
\hline \multicolumn{7}{|l|}{ Hepatomegaly } \\
\hline - Negative & 1 & $25.0 \%$ & 3 & $75.0 \%$ & \multirow{2}{*}{0.107} & \multirow{2}{*}{1.00} \\
\hline - $\quad$ Positive & 7 & $33.3 \%$ & 14 & $66.7 \%$ & & \\
\hline \multicolumn{7}{|l|}{ CNS Infiltration } \\
\hline - $\quad$ Negative & 7 & $29.2 \%$ & 17 & $70.8 \%$ & \multirow{2}{*}{2.214} & \multirow{2}{*}{0.320} \\
\hline - $\quad$ Positive & 1 & $100.0 \%$ & 0 & $0.0 \%$ & & \\
\hline \multicolumn{7}{|l|}{ Immunophenotyping } \\
\hline - $\quad$ Pre-B -ALL & 4 & $21.1 \%$ & 15 & $78.9 \%$ & \multirow{2}{*}{4.360} & \multirow{2}{*}{0.059} \\
\hline - $\quad$ mature \&progenetor B-ALL & 4 & $66.7 \%$ & 2 & $33.3 \%$ & & \\
\hline \multicolumn{7}{|l|}{ FAB } \\
\hline - L1 & 3 & $60.0 \%$ & 2 & $40.0 \%$ & \multirow{3}{*}{7.17} & \multirow{3}{*}{$0.017 *$} \\
\hline - $\quad$ L2 & 3 & $16.7 \%$ & 15 & $83.3 \%$ & & \\
\hline - $\quad$ L3 & 2 & $100.0 \%$ & 0 & $0.0 \%$ & & \\
\hline Risk & & & & & & \\
\hline - High & 8 & $61.5 \%$ & 5 & $38.5 \%$ & 10860 & ด \\
\hline - $\quad$ Standard & 0 & $0.0 \%$ & 12 & $100.0 \%$ & 10.860 & $0.002^{*}$ \\
\hline
\end{tabular}


Table (5): Relation between livin expression and laboratory findings:

\begin{tabular}{|c|c|c|c|c|c|c|}
\hline \multirow{3}{*}{ Variable } & \multicolumn{4}{|c|}{ Livin Expression } & \multirow{3}{*}{ Test $* *$} & \multirow{3}{*}{$P$ value } \\
\hline & \multicolumn{2}{|c|}{ Negative $\mathbf{N}=8$} & \multicolumn{2}{|c|}{ Positive N=17 } & & \\
\hline & No. & $\%$ & No. & $\%$ & & \\
\hline \multicolumn{7}{|l|}{ WBC } \\
\hline$<50.000 / \mathrm{mm}^{3}$ & 3 & 37.5 & 14 & 82.4 & \multirow{2}{*}{5.029} & \multirow{2}{*}{$0.025^{*}$} \\
\hline \multirow[t]{2}{*}{$\geq 50.000 / \mathrm{mm}^{3}$} & 5 & 62.5 & 3 & 17.6 & & \\
\hline & \multicolumn{2}{|c|}{ Mean \pm SD } & \multicolumn{2}{|c|}{ Mean \pm SD } & & \\
\hline $\mathrm{HB}:(\mathrm{g} / \mathrm{dl})$ & \multicolumn{2}{|c|}{$9.0 \pm 1.4$} & \multicolumn{2}{|c|}{$8.2 \pm 1.7$} & $-0.993 * *$ & 0.321 \\
\hline PLT:(x10 $\left./ \mathrm{mm}^{3}\right)$ & \multicolumn{2}{|c|}{$35.50 \pm 3.53$} & \multicolumn{2}{|c|}{$72.53 \pm 8.14$} & -1.962 & 0.091 \\
\hline PT & \multicolumn{2}{|c|}{$14.1 \pm 2.7$} & \multicolumn{2}{|c|}{$15.9 \pm 2.3$} & -0.147 & 0.883 \\
\hline PTT & \multicolumn{2}{|c|}{$29.13 \pm 3.23$} & \multicolumn{2}{|c|}{$28.59 \pm 3.12$} & $-0.444 * *$ & 0.657 \\
\hline Total bilirubin:(mg/dl) & \multicolumn{2}{|c|}{$0.8 \pm 0.2$} & \multicolumn{2}{|c|}{$0.8 \pm 0.2$} & -0.213 & 0.831 \\
\hline Direct bilirubin: $(\mathrm{mg} / \mathrm{dl})$ & \multicolumn{2}{|c|}{$0.30 \pm 0.08$} & \multicolumn{2}{|c|}{$0.30 \pm 0.04$} & -0.126 & 0.900 \\
\hline Total Protein:(g/dl) & \multicolumn{2}{|c|}{$6.7 \pm 0.6$} & \multicolumn{2}{|c|}{$6.6 \pm 0.7$} & $-0.530 * *$ & 0.596 \\
\hline Albumin(g/dl) & \multicolumn{2}{|c|}{$3.9 \pm 0.7$} & \multicolumn{2}{|c|}{$3.9 \pm 0.6$} & $-0.264 * *$ & 0.792 \\
\hline SGOT & \multicolumn{2}{|c|}{$32.3 \pm .1$} & \multicolumn{2}{|c|}{$26.8 \pm 4.9$} & $-1.547 * *$ & 0.122 \\
\hline SGPT & \multicolumn{2}{|c|}{$31.1 \pm 4.4$} & \multicolumn{2}{|c|}{$29.4 \pm 5.7$} & $-0.234 * *$ & 0.815 \\
\hline Urea(mg/dl) & \multicolumn{2}{|c|}{$12.12 \pm 1.31$} & \multicolumn{2}{|c|}{$13.44 \pm 2.19$} & $-1.321 * *$ & 0.131 \\
\hline $\mathrm{LDH}(\mathrm{U} / \mathrm{L})$ & \multicolumn{2}{|c|}{$312.0 \pm 49.0$} & & & -0.176 & 0.860 \\
\hline Creatinine (mg/dl) & & & & & -0.119 & 0.905 \\
\hline BM blast & 79 . & .81 & & .94 & -1.960 & $0.050 *$ \\
\hline Peripheral Blast & 18. & .65 & & & -0.796 & 0.426 \\
\hline
\end{tabular}

Table (6): Relation between livin expression and some prognostic factors:

\begin{tabular}{|c|c|c|c|c|c|}
\hline \multirow{2}{*}{\multicolumn{2}{|c|}{ Variable }} & \multirow{3}{*}{$\begin{array}{c}\mathbf{N}=\mathbf{1 7} \\
15 \\
2 \\
\end{array}$} & \multicolumn{3}{|c|}{ Positive Livin expression } \\
\hline & & & \multirow{2}{*}{$\begin{array}{c}\text { Mean } \pm \text { SD } \\
294.53 \pm 153.03 \\
31.0 \pm 12.73\end{array}$} & \multirow{2}{*}{$\begin{array}{r}\text { Test** } \\
-2.367\end{array}$} & \multirow{2}{*}{$\begin{array}{l}\text { P value } \\
<0.001^{*}\end{array}$} \\
\hline Age & $\begin{array}{l}<10 \\
\geq 10\end{array}$ & & & & \\
\hline \multirow[t]{2}{*}{ Sex } & Male & 7 & $284.29 \pm 184.90$ & -0.732 & 0.464 \\
\hline & Female & 10 & $249.0 \pm 163.41$ & & \\
\hline \multirow[t]{2}{*}{$\mathbf{L N}$} & Negative & 2 & $178.50 \pm 228.40$ & -0.746 & 0.456 \\
\hline & Positive & 15 & $274.87 \pm 165.19$ & & \\
\hline \multirow{2}{*}{ Splenomegaly } & Negative & 2 & $313.50 \pm 4.95$ & -0.075 & 0.941 \\
\hline & Positive & 15 & $256.87 \pm 178.27$ & & \\
\hline \multirow{2}{*}{ Hepatomegaly } & Negative & 3 & $363.00 \pm 158.75$ & -1.009 & 0.313 \\
\hline & Positive & 14 & $242.21 \pm 167.36$ & & \\
\hline WBC & $\begin{array}{l}<50.000 / \mathrm{mm}^{3} \\
\geq 50.000 / \mathrm{mm}^{3}\end{array}$ & $\begin{array}{c}14 \\
3\end{array}$ & $\begin{array}{c}249.85 \pm 155.15 \\
117.33 \pm 72.91\end{array}$ & -1.708 & 0.089 \\
\hline \multirow[b]{2}{*}{ Immunophenotyping } & Pre-B -ALL & 15 & $296.33 \pm 149.62$ & -2.237 & $0.025^{*}$ \\
\hline & Mature \& Progenetor B-ALL & 2 & $17.50 \pm 0.71$ & \multirow{3}{*}{-0.298} & \multirow{3}{*}{0.765} \\
\hline \multirow[t]{2}{*}{ FAB } & L1 & 2 & $291.00 \pm 28.28$ & & \\
\hline & $\mathrm{L} 2$ & 15 & $259.87 \pm 178.90$ & & \\
\hline \multirow{2}{*}{ Risk } & High & 5 & $82.80 \pm 131.25$ & -2.584 & $0.01 *$ \\
\hline & Standard & 12 & $338.83 \pm 116.90$ & & \\
\hline
\end{tabular}


The most frequent childhood cancer is acute lymphoblastic leukemia (ALL) ${ }^{(7)}$. Understanding of the disease's biology, customized treatment, and better supportive care have all led to an increase of rates of surviving ${ }^{(8)}$.

Cells use apoptosis, one of several types of programmed cell death (PCD), to get rid of unneeded or damaged cells. Cancer cells are known for their ability to resist apoptosis ${ }^{(3)}$.

Inhibitor of apoptosis proteins (IAPs) family belongs to the large and heterogenous group of antiapoptotic factors that are frequently over expressed in cancer cells including leukemia. As IAPs proteins also regulate several key points that control the cell's decision to live or die, they are considered to be promising targets for prognostic and therapeutic modalities ${ }^{(9)}$.

Livin is one of the IAPs family which generally interacts with caspase 3 , caspase 7 , caspase 9 , and also can inhibit SMAC/DIABLO which promotes caspases activation by binding to IAP-family members ${ }^{(5)}$.

Livin plays a dual role in tumorigenesis (antiapoptotic in full length and proapoptotic in the cleaved or truncated form ${ }^{\mathbf{( 1 0})}$.

It was found that there was no statistically significant difference between controls and cases as regard any of the tests except LDH which means show highly significant increase in cases than controls (158 \pm 17 in control group versus $310 \pm 37)(\mathrm{P}<0.001)$.

Also mean livin expression level was significantly higher in case group in comparison to control group (179.31 \pm 185.66 in control group versus 0 $.054 \pm 0.021$ in case group) $(\mathrm{P}<0.001)$.

In this study it was found that livin expression was more in age group less than 10 years ( 15 cases) than those in age group more than 10 years ( 2 cases).

This is in agreement with Ibrahim et al. ${ }^{(6)}$ who found that livin expression was more in age $<10$ years than age group $\geq 10$ years.

Livin expression might be related to the age of patients. Children, not fully matured, have a variety of different biologic features distinguishing them from adults.

Children aged 1 to $<10$ years have a more favorable outcome than infants and children aged $>10$ years this may be due to high frequency of more favorable underlying biologic features of lymphoblasts of patients of this age group ${ }^{(11)}$.

As regard sex distribution in this study it was found significant difference between negative and positive cases (livin -ve cases were 7 males and 1 female while livin $+\mathrm{Ve}$ cases were 7 males and 10 females.

Choi et al. ${ }^{(12)}$, found livin expression was higher in females $33 \%$ of females expressed livin while $21 \%$ of males expressed livin. While Ibrahim et al. ${ }^{(6)}$ found no significant difference as regard sex between negative and positive cases (12 males and 15 females versus 27 males and 26 females).
Like many other diseases and cancers, childhood ALL risk shows consistent sex differences. Relapse and secondary malignancies are more common in males this may be due to the occurrence of testicular relapse ${ }^{(\mathbf{1 3})}$.

As regard WBCs it was found that in livin negative subgroup ( 8 cases): there were 3 patients $(37.5 \%)$ with leucocytic count $<50.000 / \mathrm{mm}^{3}$ at diagnosis and 5 patients $(62.5 \%)$ with leucocytic count $\geq 50.000 / \mathrm{mm}^{3}$ or more at diagnosis. Compared to 14 $(82.4 \%)$ and 3 patients $(17.6 \%)$ among livin positive group (17 cases) respectively ( $\mathrm{p}=0.025)$.

As WBCs count at diagnosis is an adverse prognostic factor ${ }^{(\mathbf{1 4})}$ so, it could be concluded that livin may be associated with good prognosis of childhood acute lymphoblastic leukemia.

This was in agreement with Ibrahim et al. ${ }^{(6)}$ who found in livin negative group (27 cases) 10 patients with leucocytic count $<50.000 / \mathrm{mm}^{3}$ at diagnosis and 17 patients with leucocytic count $\geq 50.000 / \mathrm{mm}^{3}$ or more at diagnosis. Compared to 45 and 8 patients among livin positive group (53 cases) respectively $(\mathrm{p}<0.001)$.

In this study it was found that livin expression was significantly higher in standard risk group patients than high risk group patients. Mean livin expression in standard risk group was $338.83 \pm 116.90$ while in highrisk group was $82.8 \pm 131.25$.

This was in agreement with Ibrahim et al. ${ }^{(6)}$ who found mean livin expression level in standard risk group was $128.13(0.84-519.1)$ versus 8.6 (4.6-110.6) in high-risk group.

As regard B.M blast it was found that there was significant difference in B.M blast between livin negative cases and livin positive case. B.M blast was significantly higher in livin negative subgroup. (Mean \pm SD of B.M blast in livin negative and livin positive cases was79.75 \pm 9.81 and $68.65 \pm 12.94$ respectively).

This was in agreement with Zareifar et al. ${ }^{(15)}$ who found that in livin positive group ( 35 patients) there were 6 patients with blast $<50 \%$ and 29 patients with blasts $>50 \%$, while in livin negative group ( 8 patients) there were 2 patients with blasts $<50 \%$ and 6 patients with blasts $>50 \%$.

Choi et al. ${ }^{(12)}$, demonstrated that the existence of positive prognostic variables at diagnosis was, although not always, related with Livin expression in childhood ALL. Furthermore, a positive early response to chemotherapy was linked to high levels of Livin expression. In addition, it was found that Livin expression rates and livin expression levels were higher in patients with favorable clinical aspects compared to those in patients with negative clinical features.

Positive correlation between livin and good prognostic factors of childhood ALL may be explained by evidence that leukemic cells contain the cleaved form of livin and expressed Livin. In other words, this cleaved version hasn't been tested in other types of 
cancer ${ }^{(16)}$. Perhaps the cleaved form possessed proapoptotic properties ${ }^{(15)}$.

The results of the current study are also in contrast with El-Mesallamy et $\boldsymbol{a l}$. ${ }^{(17)}$ as they revealed the existence of poor prognostic variables at diagnosis was linked to elevated livin expression in ALL patients. High livin expression was also linked to a decreased EFS and OS in both groups of participants. Leukemia cells that produce high levels of livin are less responsive to chemotherapeutic agent-induced apoptosis.

Also, Zareifar et al. (15), found that Livin showed significant associations with some poor prognostic factors of childhood leukemia.

The controversy between the previous results and our results may be explained by Abd-Elrahman $\boldsymbol{e t}$ al. (10) and Lazar et al. (18) who observed the proapoptotic activity of truncated form of livin (t-livin) which is more predominant in children ${ }^{(6)}$.

\section{CONCLUSION}

It could be concluded that livin was associated with favorable prognostic factors among ALL patients: (age $<10$ years, female patients, WBCs $<50,000 / \mathrm{mm}^{3}$ ).

Financial support and sponsorship: Nil.

Conflict of interest: Nil.

\section{REFERENCES}

1. Paul S, Kantarjian H, Jabbour E (2016): Adult Acute Lymphoblastic Leukemia. Mayo Clin Proc., 91(11):1645-1666.

2. Chen L, Zeng Y, Zhou S (2018): Role of Apoptosis in Cancer Resistance to Chemotherapy. Intech Open, 18. https://www.intechopen.com/chapters/59686

3. Cong $\mathrm{H}, \mathrm{Xu} \mathrm{L}, \mathrm{Wu}$ Y et al. (2019): Inhibitor of Apoptosis Protein (IAP) Antagonists in Anticancer Agent Discovery: Current Status and Perspectives. J Med Chem., 62(12):5750-5772.

4. Barbara A, Silviu S, Silvia D et al. (2017): Livin /BIRC7 expression as malignancy marker in adrenocortical tumours. Oncotarget., 8: 9323-9338.

5. Yan B (2011): Research progress on Livin protein: an inhibitor of apoptosis. Mol Cell Biochem., 357(12):39-45.
6. Ibrahim A, Khaled H, Ikhail N et al. (2014): Cancer incidence in Egypt: Results of the national population bases cancer registery program. Journal of Cancer Epidemiology, 14: 437-971.

7. Iacobucci I, Mullighan C (2017): Genetic basis of acute lymphoblastic leukemia. J Clin Oncol., 35 (9):975-983.

8. Siegel R, Miller K, Jemal A (2019). Cancer statistics, 2019. Cancer Journal for Clinicians, 69(1): 7-34.

9. Fulda $S$ (2014): Inhibitor of apoptosis (IAP) proteins in haematological malignancies: molecular mechanisms and therapeutic opportunities. Leukemia, 28: 14141422.

10. Abd-Elrahman I, Hershko K, Neuman T et al. (2009): The inhibitor of apoptosis protein Livin (MLIAP) plays a dual role in tumorigenicity. Cancer Res., 69(13):5475-5480.

11. Forestier E, Schmiegelow K (2006): The incidence peak of the childhood acute leukemia reflect specific cytogenic aberration. J Pediatric Haematol Oncol., 28 (8):486-495.

12. Choi J, Hwang Y, Sung K et al. (2007): Expression of livin, an anti-apoptotic protein, is an independent favorable prognostic factor in childhood acute lymphoblastic leukemia. Blood, 109(2):471-477.

13. Belson M, Kingsley B, Holmes A (2007). Risk factors for acute leukemia in children: a review. Environmental Health Perspectives, 115(1): 138-145.

14. Lee J, Cho B (2017): Prognostic factors and treatment of pediatric acute lymphoblastic leukemia. Korea J Pediatr., 60(5):129-137.

15. Zareifar S, Ghorbani S, Monabbati A et al. (2018): Expression of antiapoptotic proteins livin and survivin in pediatric AML patients, as prognostic markers. Pediatr Hematol Oncol., 35(4):250-256.

16. Rigato D, Branco $P$, Silva $C$ et al. (2020): BIRC7 (baculoviral IAP repeat containing 7). Atlas of Genetics and Cytogenetics in Oncology and Haematology, 20: 24-30.

17. El-Mesallamy H, Hegab H, Kamal A (2011): Expression of inhibitor of apoptosis protein (IAP) livin/BIRC7 in acute leukemia in adults: correlation with prognostic factors and outcome. Leuk Res., 35(12):1616-22.

18. Lazar I, Perlman R, Lotem M et al. (2012): The clinical effect of the inhibitor of apopotosis protein livin in melanoma. Oncology, 82(4): 204-209. 